\title{
Modular building of formative systems for machine tools structures
}

\author{
G. Todorov, C. Peychev, N. Todorov \\ Technical University Sofia, Dept. TMMM, \\ Sofia - 1156, Bulgaria, tel: (+359) (2) 6362574 \\ e-mail:gdt@alpha.vmei.acad.bg
}

\begin{abstract}
The purpose of the present work is to create a systematic approach to effective modular building by synthesis of numerous variants of formative systems (FS) for machine-tools (MT). On the basis of the necessary formative motions, generalized geometry and structure of the elements of FS as well as the variety of its building, a minimum needed and sufficient number of basic structure elements (BSE) is defined. By these BSE all of the kinematics and configurative variants of structures of FS could be built. Thus a library of modules is differentiated. A coded description of BSE is created with a view to be established rules for their connection and automated generation of structures of FS.
\end{abstract}

Key words

CAD, virtual design, modular building, machine tools

\section{INTRODUCTION}

Producers of MT are faced with the necessity of showing a resourceful adaptation to the market demand, because of its continuously changeable conditions and growing requirements to the modern machine-building production. Such a principle involves building of MT, which largely satisfy specific requirements on the basis of a limited number of functionally differentiated and completely 
designed elements, called modules, in short terms of delivery and foreseeing working time.

The problems related to modular building of MT are object of a set of publications (Lijbenov, 1990), (Averianov, 1987), (Ito and Shino, 1982), ets. Systems for modular building consist of two mutually connected parts. The main part of such kind of systems refers to the financial provision, which includes a distinct nomenclature of modules. The other important part is the logical provision that actually is a combination of rules.

From already mentioned theoretical investigations and practical realizations of modular systems ( Rapid, Modu-Lina, Omnimil, Ekokut - 2, Over, Transfer Center, Mazak, ets. ) there could be sharply outlined the following basic states which describe the modular principle, grounded within these systems:

- the limited nomenclature of modules must afford a possibility for creation of a variety of configurations of MT through modular combinations and various situations and connections of modules;

- the modular principle of design, largely satisfies the requirements for effective solution of a concrete technological task;

- the time and labor costs of design process is shortened;

- the working reliability of MT is increased at the expense of the working off of the modules contained in it.

- the adaptation degree of a given modular configuration to the running technological process is maximized.

The subject of the present work is to create a systematic approach to an effective modular building of FS. In particular, it means building of FS with various functions and purposes by a minimum number of building elements, named modules.

\section{THEORETICAL PRINCIPLES}

Modular building requires analysis of all the technological, kinematics and configuration variants. The main questions: how the single modules are differentiated, what has to be the minimum number of modules necessary and sufficient for the building of FS with various functions, what kind of principles are necessary for the generation and estimation of the various variants, ets.; are fundamental in a synthesis method.

\subsection{Necessary formative motions}

The following must be taken into account (Lijbenov, 1990) under the determination of the necessary formative motions:

- for a solid (surface) to be worked up, the working tool must be able to reach every point of the surface; 
- in most cases it is necessary for the metal-cutting tool to have an accurately fixed orientation towards the processed surface;

- this orientation must be maintained constant or must be changed according to a definite rule during the machining.

The six degrees of freedom (DOF) of a solid in the space could be divided into three position DOF necessary for the reaching of every point of the processed surface and three orientation DOF necessary to enable the orientation of the tool towards the surface in the working process. Some of the motions ( for example, main rotational motion of the tool under cutting process) have technological character and don't pertain to the formative motions. Position motions, enabled by the FS can be rotational as well as translation, but orientation motions can only be rotational. The motions of the FS will be marked as $\mathrm{P}$ and $\mathrm{Q}$ - for position and orientation motions respectively.

\subsection{Technological modules}

These modules are functionally and constructively differentiated elements of the FS, which bear the tool or the detail. They can realize the cutting support motion through their own operation that is non formative. These modules can be attached to the rest of the BSE and they belong to the FS, but they are not a part of its FS kinematics structure. The technological modules, that bear the tool are reduced to a module for tool without, Fig.1.a. and with, Fig. 1.b, rotational technological motion. The technological modules which bear the detail are a module for prismatic - corps details, Fig. 1.c and module for rotational details, Fig. 1.d.

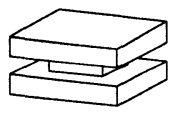

a)

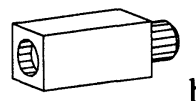

b)

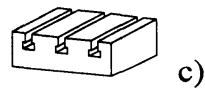

c)

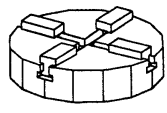

d)

Figure 1 Technological modules.

\subsection{Virtual variety of FS structure}

The necessary number of DOF as well as their kinematics description must be defined, (Mavroidis and Roth, 1994). The FS must possess these DOF so that they will be able to process one or several of the geometrical-kinematic forms with the appropriate kind of tool. Thus, according to the selected tool, the processed surface and the position of the tool towards this surface (technological scheme) determines the first level of variety in the structural building of the FS of MT when the 
technological equivalence is preserved. This first level is called technological variety.

\section{Technological variety.}

This variety defines the necessary DOF for FS according to the kind of the processed surface, selected tool and selected technological scheme (Lijbenov, 1990) . The number of position motions is marked as 1P, 2P and 3P. The number of orientation motions is marked as $1 \mathrm{Q}, 2 \mathrm{Q}$ and $3 \mathrm{Q}$. When there are i-positional and $\mathrm{k}$-orientation motions necessary for the orientation of the tool in a given position, the expression is $\mathrm{P}+\mathrm{kQ}$.

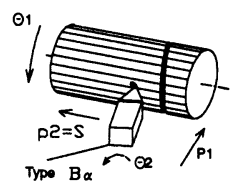

a)

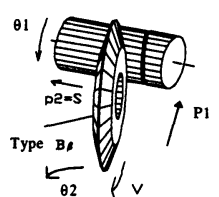

b)

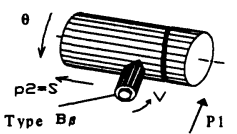

c)

Figure 2 Technological variety.

As an example for technological variety, there could be examined a machining of a helical groove with variable pitch - Fig.2. The machining by lathe-tool Fig.2.a., requires $2 \mathrm{P}+2 \mathrm{Q}$ motions necessary for the $\mathrm{FS}$, the mill in Fig.2.b. requires $2 \mathrm{P}+2 \mathrm{Q}$ motions (there must be an orientation of the axis of the mill towards the axis of the processed surface). When milling by perpendicular to the axis cotter drill Fig.2.c. $2 \mathrm{P}+1 \mathrm{Q}$ motions are necessary.

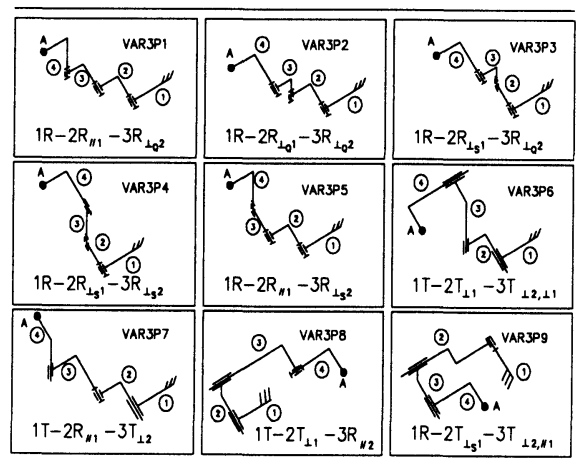

Figure 3 Kinematic structure - 3p variants.

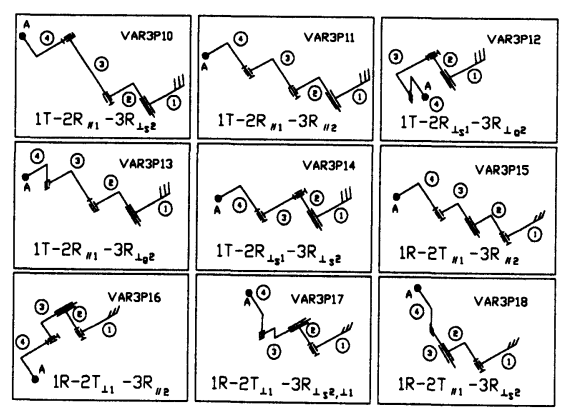




\section{Kinematic variety}

According to the necessary position motions a kinematic variety is formed. It develops into two varieties. The first variety is related entirely to the position motions and especially to the method of kinematic realization of the necessary number of DOF by the use of rotational and translation kinematic pairs as well as their mutual disposition in the space. Only the case of orthogonal position of the kinematic pairs is considered. Thus all possible mutual positions of the motions are covered because of the accepted orthogonality of the kinematic pairs. The number of the variants in the first kinematic variety is considerable: in one position motion there is only one translation; in two position motions their number gets to four variants and in three position motions their number gets to eighteen structuralkinematic variants as shown in Fig. 3.

For instance, in Fig.4 there are two variants for realization of the necessary motions of the drilling machine. Three position motions are necessary. For their realization three mutually perpendicular translations can be used (var. 3P6 from Fig.3) as it is shown in Fig. 4.a. The three translational motions also can be realized only by rotations (var. 3P5 from Fig.3) as it's shown in Fig. 4.b.
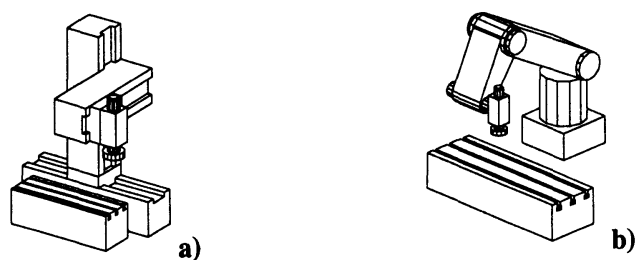

Figure 4 3p kinematic variants - example by co-ordinate drilling machine.

The second kinematic variety is determined by the mutual position (place and sequence) of the $\mathrm{P}$ and $\mathrm{Q}$ - motions in the structure. This variety is divided to two sublevels:

- At the first sublevel the variety evolves from the place of the Q-motions towards position ones, fig.5. This table shows that the motions for orientation couldn't be concentrated one to the other and it gives all the possible places of the $Q$ towards $\mathrm{P}$-motions in cases of one, two and three orientation motions. This division of the motions is conditional and there is a possibility of penetration of the motions. Generally, the formative system must not be changed functionally and kinematically and at the same time the penetration of the motions from Qstructure to P-structure must be allowed. All this results in the following limitation: Q- motion and P-motion can exchange their places in the structure of the formative system only if they are along or around the same axis. It comes to 
show that penetration of the motions is possible only when a rotational from the Q-motions (and they are only rotational) has an axis of rotation, which is parallel to the translations, P- motion. In this case only a rotation from Q- motions can be replaced by a translation from $\mathrm{P}$ - motions.

- At the second sublevel the variety finds expression in the segmentation of motions along the same axis. This stage of the second variety of the kinematic variety relates predominantly to the telescopic solutions.

\begin{tabular}{lllll}
\hline Number of "Q" & \multicolumn{4}{l}{ Kinematic variants } \\
& $1 \mathrm{Q} / \mathrm{P} /$ & $/ \mathrm{P} / 1 \mathrm{Q}$ & & \\
\hline 1 & $2 \mathrm{Q} / \mathrm{P} /$ & $1 \mathrm{Q} / \mathrm{P} / \mathrm{Q}$ & $/ \mathrm{P} / 2 \mathrm{Q}$ & \\
\hline 2 & $3 \mathrm{Q} / \mathrm{P} /$ & $2 \mathrm{Q} / \mathrm{P} / 1 \mathrm{Q}$ & $1 \mathrm{Q} / \mathrm{P} / 2 \mathrm{Q}$ & $/ \mathrm{P} / 3 \mathrm{Q}$ \\
\hline 3 & & &
\end{tabular}

Figure 5 Second level of kinematic variants

\section{Configurative and conceptual variety.}

The variety of the building one towards another of the elements is represented mainly by the kinematic variants, but not quite. The configurative variants in terms of left and right configuration, which usually are functionally identical, appear to be a further possibility for development of the variety under the building of FS. The configurative level of variety in reference to the orientation of the formative system towards the static basic coordinates is determined by two typical moments that represent two sublevels:

- The first sublevel is determined by the elements which are fixed in reference to the static coordinates and it is reduced to the selection of a basic (connected immovably to the seating) element. The number of variants is equal to the number of BSE, which take part in realization of the motion, plus two, giving an account of the technological modules, carrying the detail and the tool. It's possible between two next situated elements in the structure not to be realized a motion, too. An example for the sublevel of the configurative variety in reference to the fixed element are the various configurations of the milling machines as shown in Fig. 6. The number of configuration variants according to the stopped (basic) module is four.

- The second sublevel is in the choice of the spatial orientation of the detail or the tool, i. e., it determines the axis of rotation or the direction of moving of the main cutting motion. The possibilities are two: vertical or horizontal configuration. Intermediate variants are possible, too. Mainly, the kind of the configuration is determined by the form and the size of the details. 


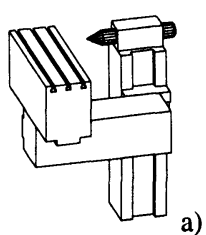

a)

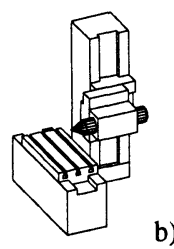

b)

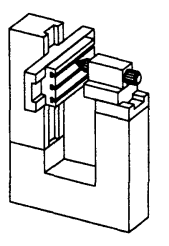

c)

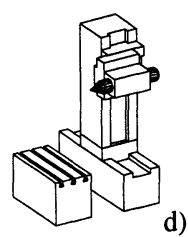

Figure 6 First level of Configurative and conceptual variety.

\section{Constructive variety}

The fourth level of variety is the so-called constructive variety. It refers to the concrete constructions of the formative system and respectively the number of variants is immense. The last two levels, in that point of the paper, won't be considered in detail.

\subsection{Theoretical conclusions}

Up to here the following conclusions could be made:

- the technological modules, which bear the tools and the details and don't pertain to the kinematics structure of the formative system, are defined;

- technological, kinematics and basic configuration variants with generalized geometry under structural building of MT are pointed;

- it's necessary to create a minimum necessary and sufficient number of BSE by which the building of all structure types is possible.

\section{SYNTHESIS OF FS}

The single modules must be defined, coded for computer processing and united in a common library. The rules for realization of the connections between the modules must be formulated. When all of that is done it would be possible to go to the first stage of the automated design: synthesis of FS with various stages of participation during this synthesis on behalf of the designer (the so called interactive and automated generation of FS).

\section{GENERATION OF MODULES LIBRARY}

Every machine-tool can be considered as a system, consisting of a great number of constructive elements fitted together by their surfaces of joint. Under MT constructed by using modular principles these elements are constructively independent of each other. They are united in a library of BSE having the ability to 
combine each other under appropriate rules, guaranteeing the functionality of the MT. The modular library has to include minimum necessary and sufficient number of elements for the building of every FS of machine-tool, which is required for the production of a particular type of detail.

\subsection{Definition of the minimum necessary number of BSE}

The number of BSE in the library is determined by the motions, that have to enable their mutual situation in the single modules and also by the constructive signs describing the elements.

\section{Necessary motions and their mutual location}

The needed motions, which must be provided by the elements in FS are translational, marked as "T", Fig. 7.a., Fig. 7.b.; rotational, marked as "R", Fig. 7.c., Fig. 7.d.;fixed stick, which enables fixed joint between the elements, marked as "N" Fig. 7.e.
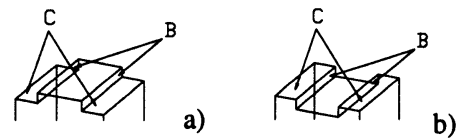

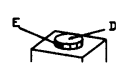

c)

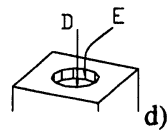

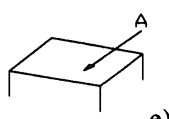

e)

Figure 7 Types of sticks.

The stick surface, which enables the joining of the element to a transitional element of the structure is called "entrance". The stick surface, which enables the joining of the element to the next element of the structure is called "exit". The kinematic joints between the sections have only one degree of freedom and since a stick surface represents exactly one kinematic joint, every stick enables only one or not a single motion. The motions, provided by the element through its entrance are called "entrance" for that element and that ones that are provided through the exit are called "exit". All fixed sticks are featured as flat surfaces Fig. 7.e. The translational entrance is a convex prismatic guide, Fig. 7.a. and rotational entrance is a convex short cylinder, Fig. 7.c. The translational exit surface is represented by a concave prismatic guide Fig. 7.b. and rotational exit surface - as a concave short cylinder Fig. 7.d. A right oriented co-ordinate system is defined for every stick according to certain rules as shown in Fig. 8.

Because of the accepted orthogonal mutual situation of the kinematic connections, all the possible mutual situations of the exit towards the entrance of the same element are:

- Perpendicularity with intersection of the axes, marked as "X".

-Perpendicularity without intersection of the axes marked as "Q". 
-Parallelism of the axes without coincidence marked as "I". When a stick is fixed, the mutual situation of the exit towards the entrance is marked as "I". Coincidence of the axes of the motions is marked as " $\mathrm{C}$ ".
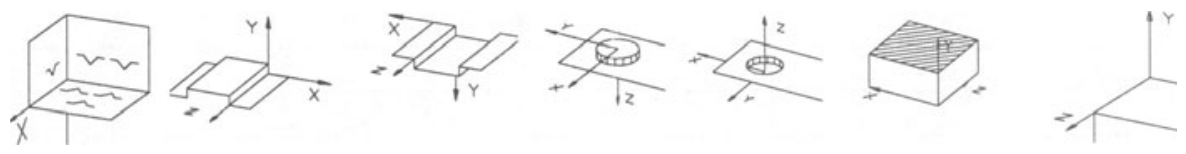

Figure 8 Coordinate systems.

Table 1 First level of differentiation of BSE

\begin{tabular}{lllllllllllll}
\hline No & 1 & 2 & 3 & 4 & 5 & 6 & 7 & 8 & 9 & 10 & 11 & 12 \\
\hline BSE & $\mathrm{N}$ & $\mathrm{N}$ & $\mathrm{T}$ & $\mathrm{N}$ & $\mathrm{R}$ & $\mathrm{R}$ & $\mathrm{T}$ & $\mathrm{T}$ & $\mathrm{T}$ & $\mathrm{T}$ & $\mathrm{R}$ & $\mathrm{R}$ \\
& $\mathrm{I}$ & $\mathrm{I}$ & $\mathrm{Q}$ & $\mathrm{I}$ & $\mathrm{X}$ & $\mathrm{Q}$ & $\mathrm{I}$ & $\mathrm{X}$ & $\mathrm{Q}$ & $\mathrm{I}$ & $\mathrm{I}$ & $\mathrm{C}$ \\
& $\mathrm{N}$ & $\mathrm{T}$ & $\mathrm{T}$ & $\mathrm{R}$ & $\mathrm{R}$ & $\mathrm{R}$ & $\mathrm{R}$ & $\mathrm{R}$ & $\mathrm{R}$ & $\mathrm{T}$ & $\mathrm{R}$ & $\mathrm{R}$ \\
\hline BSE & & $\mathrm{T}$ & & $\mathrm{R}$ & & & $\mathrm{R}$ & $\mathrm{R}$ & $\mathrm{R}$ & & & \\
Inverted & & $\mathrm{I}$ & & $\mathrm{I}$ & & & $\mathrm{I}$ & $\mathrm{X}$ & $\mathrm{Q}$ & & & \\
& & $\mathrm{N}$ & & $\mathrm{N}$ & & & $\mathrm{T}$ & $\mathrm{T}$ & $\mathrm{T}$ & & & \\
\hline
\end{tabular}

The type of the entrance and exit, enabled by the two stick surfaces and the mutual situation of their axes lead to twelve varieties of BSE, Table 1. When there are exit and entrance of the opposite charge the second type of BSE with inverted (Raghavan and Roth, 1992/1993) exit and entrance must be added for the provision of full ability for sticking between the elements. This way the number of varieties amounts to 17 . The BSE could be reduced to three types: boxes, marked as "C", beams, marked as "B" and plates, marked as "P" according to the relative proportions of height, length and width. By combining the three types of BSE with the variants of Table 1. Table 2. is done. In this table by " 2 " is marked the existence of two BSE of the given type, by " 1 " is marked the existence of one BSE, and with "0" - the lack of BSE. Thus, 43 BS E come out. Further, these 43 BSE are worked out in detail, which leads to augmentation of the virtual number of BSE in the library. However, these 43 BSE represent the minimum necessary number of elements for building all of the technological, kinematic and configurative variants from the previous chapter. 
Table 2 Second level of differentiation of BSE

\begin{tabular}{lllllllllllll}
\hline No & 1 & 2 & 3 & 4 & 5 & 6 & 7 & 8 & 9 & 10 & 11 & 12 \\
\hline Entrance & $\mathrm{N}$ & $\mathrm{T}$ & $\mathrm{T}$ & $\mathrm{R}$ & $\mathrm{R}$ & $\mathrm{R}$ & $\mathrm{R}$ & $\mathrm{R}$ & $\mathrm{R}$ & $\mathrm{T}$ & $\mathrm{R}$ & $\mathrm{R}$ \\
\hline Entr. Disp. & $\mathrm{I}$ & $\mathrm{I}$ & $\mathrm{Q}$ & $\mathrm{I}$ & $\mathrm{X}$ & $\mathrm{Q}$ & $\mathrm{I}$ & $\mathrm{X}$ & $\mathrm{Q}$ & $\mathrm{I}$ & $\mathrm{I}$ & $\mathrm{C}$ \\
\hline Exit & $\mathrm{N}$ & $\mathrm{N}$ & $\mathrm{T}$ & $\mathrm{N}$ & $\mathrm{R}$ & $\mathrm{R}$ & $\mathrm{T}$ & $\mathrm{T}$ & $\mathrm{T}$ & $\mathrm{T}$ & $\mathrm{R}$ & $\mathrm{R}$ \\
\hline Boxes (C) & 1 & 2 & 1 & 2 & 1 & 0 & 2 & 2 & 2 & 1 & 1 & 1 \\
\hline Beams (B) & 1 & 2 & 1 & 2 & 1 & 1 & 2 & 2 & 2 & 1 & 1 & 1 \\
\hline Plates (P) & 1 & 2 & 1 & 2 & 0 & 0 & 0 & 2 & 0 & 1 & 0 & 1 \\
\hline
\end{tabular}

Determination of the minimum number of BSE in the library.

For the various BSE - boxes, beams and plates a full combination of entrance and exit motions and their mutual spatial situation is done observing that the entrance stick surfaces are convex, and the exit stick surfaces are concave as well as the replacement of exits and entrances of opposite charge is performed through the full combinations of entrance and exit motions. When the various entrances and exits are situated on the facets of boxes certain rules are observed, some of which are:

A. The edges of the boxes are commensurable, which leads to equivalence of all facets and edges when situating the entrance stick.

B. After fixing the entrance, exactly 5 surfaces of the element remain vacant for situation of the exit.

C. "Significant surface", means the facet of the element, upon which it is reasonably a stick to be put. When there is an exit of type rotation, all facets adjacent to the entrance rotational stick surface are equivalent, in regard to the exit (A,B,C,D) Fig. 9.a. Therefore "significant", in regard to the exit are two facets one of the adjacent to the entrance (for instance D) and the opposite (surface E) to that entrance. When there is an entrance of prismatic type or of type fixed stick, there aren't any restrictions to the situation of the exit, in regard to the other facets of the element. "Significant" for the exit are 5 surfaces (A,B,C,D,E), Fig. 9.b.

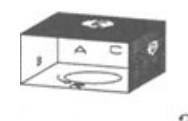

a)

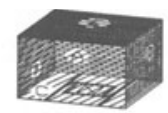

b)
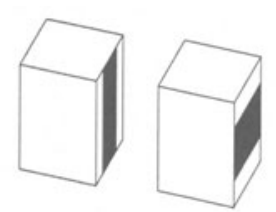

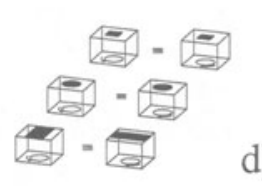

c)

Figure 9 Disposition of the sticks on boxes 
D. The type of the exit determines the cases of its situation towards the edges of the facet, upon which it is placed. If there is a prismatic exit, it's important how it is oriented towards the edges of the facet it lies on Figure 9.c.

E. An intersection between prismatic sticks is not allowed.

F. In case of rotational entrance and exit situated on the opposite facet of that entrance, the orientation of the exit towards the edges of the facet, upon which is situated this exit, doesn't matter Fig. 9.d.

In a similar way as the boxes, the rules for the plates and the beams are defined. Fig. 10 presents the table in a graphic form of the above defined rules for the boxes, the plates and the beams.

\subsection{Composing the library of BSE and FS building through it}

On the basis of the above mentioned combinations of entrance and exit motions, their spatial mutual disposition, the generalized geometry of the bed frame elements and according to the Table 2 the necessary BSE are put into the library. This library consists of 75 BSE. Every type of FS could be built by these BSE. All of the elements are identified using unique 8-symbol code, comprising all the information necessary for computer processing.

\section{CONCLUSION}

A system method for modular synthesis of FS of MT is created, including theoretical, methodical and other aspects in the process of design of MT. In this method dominates the technological function, kinematic realization and configurative variety when defining the minimum necessary and sufficient number of BSE as well as variant structures of FS on this basis. The following conclusions of greater importance can be made:

- Technological, kinematic and basic configurative variants in structural building of MT are analyzed considering generalized sizes of the elements.

- On the basis of the theoretical analysis of the necessary formative motions, the generalized geometry of the elements, building the structure of the FS and the variety of its building, a minimum necessary and sufficient number of BSE is defined. On this basis a library of modules is created. 


\begin{tabular}{|c|c|c|c|c|c|c|}
\hline \multirow[t]{2}{*}{$N$} & \multirow[t]{2}{*}{ In } & \multirow[t]{2}{*}{ Out } & \multirow[t]{2}{*}{$B O X E S$} & \multirow[t]{2}{*}{ PLATES } & \multicolumn{2}{|l|}{$B E A M S$} \\
\hline & & & & & & input along wall \\
\hline 1 & $\mathrm{R}$ & $\mathbf{R}$ & (8) & 8 & $\prod_{1}^{\infty}$ & $\sum_{i=0}^{\infty}$ \\
\hline 2 & $R$ & $\mathrm{~T}$ & -6 & 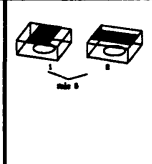 & & 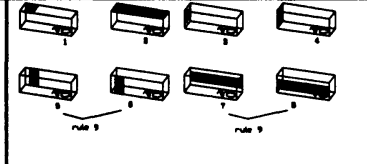 \\
\hline 3 & $\mathbf{R}$ & $\mathrm{N}$ & ही, & ES & & 在 \\
\hline 4 & $\mathrm{~T}$ & $R$ & 照 & $\mathbb{E}_{1}$ & (1) & 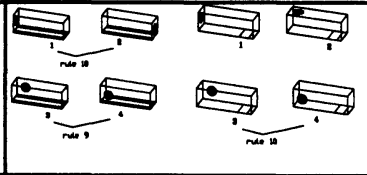 \\
\hline 5 & $T$ & $\mathrm{~T}$ & $\underbrace{90}_{0}$ & & & 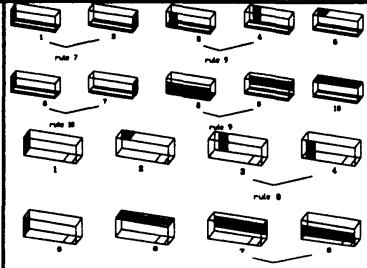 \\
\hline 6 & $T$ & $\mathrm{~N}$ & 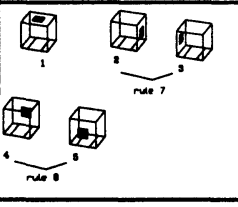 & & (1) & $\underset{\infty}{\approx} \approx$ \\
\hline 7 & $\mathbf{N}$ & $\mathrm{R}$ & $\underbrace{\infty}_{\infty}$ & & $\prod_{-\infty}^{\infty}$ & $\underset{10}{\infty}$ \\
\hline 8 & $\mathrm{~N}$ & $T$ & 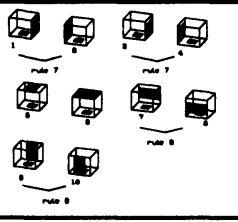 & 舟 & 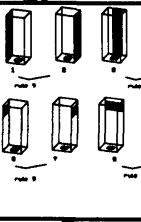 & 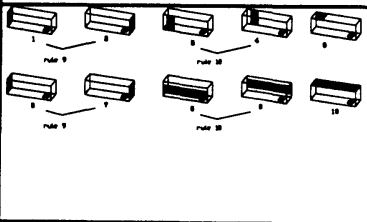 \\
\hline 9 & $N$ & $\mathbf{N}$ & 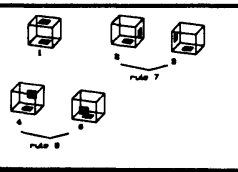 & & II) & 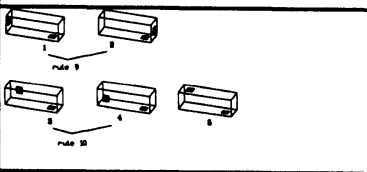 \\
\hline
\end{tabular}

Figure 10 Library of BSE. 
- A coded description of the BSE in the library is created with a view to make rules for their joining and automated generation of structures of FS of MT.

\section{Further steps}

- A system for interactive and automated synthesis of technologically equivalent FS, built through the library of BSE must be created.

- Criteria and rules for assessment of the synthesized variants of FS must be defined so that an optimal choice for certain conditions of production will be possible.

\section{REFERENCES}

Averianov, O. (1987) Module principle of CNC machine tools building. Machinostroene, Moscow.

Ito, Y., and Shino, H. (1982) Structural description and similarity evaluation of the structural configuration in machine tools, Int. J. Mach. Tool Des. Res. Vol. 22, No 2.

Lijbenov, Al. (1990) Systematic method for machine tools structure building. Machinostroene, 391 , Sofia.

Mavroidis C. and Roth B. (1994) Structural Parameters Which Reduce the Numbers of Manipulator Configuration - ASME Journal of Mechanical Design, vol. 116/11, March.

Raghavan M. and Roth B. ( 1992) A General Solution for the Inverse Kinematics of All Series Chains - Proceedings of the 8th CISM-IFT Symposium on Robots and Manipulators- Cracow, Poland.

Raghavan M. and Roth B. (1993) Inverse Kinematics of the General 6R Manipulator and Related Linkages - ASME Journal of Mechanical Design, vol. 115, No. 3 .

\section{BIOGRAPHY}

Ass. Prof. Ph.D. MSc. GEORGE TODOROV: Born in 1961, MSc. Mech. Eng., 1986, T U - Sofia, MSc. Applied Mathematics, 1988, T U - Sofia. Ass. Professor in T U - Sofia since 1987. Head of Laboratory "CAD/CAM in Industry", since 1992. Member of Bulgarian Scientific Union-NTS.

MSc. CHRISTO PEYCHEV: Born in 1970, MSc. Mech. Eng. 1994, T U - Sofia. Project Manager, since 1996, NABLA SOFTWARE Ltd. , Sofia

Prof. Ph.D. MSc. NIKOLA TODOROV: Born in 1936, MSc. Mech. Eng. 1960, T U - Sofia, Ph.D. 1968. Professor in T U - Sofia since 1980. Member of IFIP, WG 